\title{
Grain Quality and Yield of Rice in the Main and Ratoon Harvests in the Southern U.S.
}

\author{
Haiya Cai ${ }^{1,2, \dagger}$, Rodante E. Tabien ${ }^{2, \dagger}$, Deze Xu ${ }^{1 \dagger}$, Chersty L. Harper ${ }^{2}$, Jason Samford ${ }^{3}$, Yuanyuan Yang ${ }^{1}$, \\ Aiqing You ${ }^{1}$, Stanley Omar P. B. Samonte ${ }^{2}$, Leon Holgate ${ }^{2} \&$ Chunhai Jiao $^{1}$ \\ ${ }^{1}$ Food Crops Institute, Hubei Academy of Agricultural Sciences, Wuhan, China \\ ${ }^{2}$ Texas A\&M AgriLife Research Center, Beaumont, Texas, USA \\ ${ }^{3}$ Texas A\&M AgriLife Research, Wintermann Rice Research Station, Texas, USA \\ Correspondence: Chunhai Jiao, Food Crops Institute, Hubei Academy of Agricultural Sciences, Wuhan, 430064, \\ China. Tel: 86-1398-6152-279. E-mail: jiaoch@hotmail.com
}

Received: June 5, 2019

Accepted: July 12, 2019 Online Published: September 15, 2019

doi:10.5539/jas.v11n15p1

URL: https://doi.org/10.5539/jas.v11n15p1

\section{$\dagger$ These authors contributed equally to this work.}

\begin{abstract}
The ratoon rice system is an energy-saving, high-efficiency cultivation method. Harvests from a two-year field trial with a main crop (MC) and a ratoon crop (RC) were used to evaluate milled grain quality traits and yield performance. The results indicated that chalkiness was significantly lower in the RC than in the MC. Chalkiness ranged from 1.90 to $15.01 \%$, with an average of $6.46 \%$, in the $\mathrm{MC}$ and from 0.66 to $3.28 \%$, with an average of $1.50 \%$, in the RC across two years. In addition, nearly all of the RC of the test entries had lower white vitreous (higher translucency) than the MC of the same entry. In 6 of the 20 entries, the MC had longer or wider milled grain than the $\mathrm{RC}$ in 2017. The milled rice recovery for the $\mathrm{MC}$ was higher in both years, but there was no difference in head rice recovery within the same year. The average total yield $(\mathrm{MC}+\mathrm{RC})$ in the two years was 12.6 and 13.0 t/ha, and the two-year average RC yields were 47.5 and $37.3 \%$ those of the MC. Our results revealed that the RC milled grains showed better appearance quality than the MC grains, and several genotypes had comparable or even better milled grain quality and yield compared with the check entries that were suitable for the ratoon rice system.
\end{abstract}

Keywords: grain yield, milled rice, ratoon, grain quality

\section{Introduction}

As one of the most important staple foods, rice feeds nearly half of the population in the world. However, with increasing human population and decreasing arable land, rice yield must be doubled by 2050 to meet food demand (Nathaniel et al., 2012). There are two ways to improve crop yield: increase the yield per unit of land (Cassman et al., 2003) or harvest the existing cropland more frequently (Ray et al., 2013). For the former strategy, crop yields have experienced plateaus in recent years (Grassini et al., 2013), and for the latter, ratoon rice would be one of the choices (Chen et al., 2018). The ratoon crop (RC) is the second harvest from the tillers originating from the stubble of the previous crop, known as the main crop (MC); the growth duration of the RC is shorter, approximately 40\% shorter than the 100-120 days required for the MC (Krishnamurthy et al., 1988). For this reason, it is suitable for areas where the annual accumulation of solar radiation and temperature is in excess of the requirements for a single season but not enough for a double season. In addition, a ratoon crop saves seed costs and approximately $50 \%$ of labor and $60 \%$ of water costs because it is free of land preparation, seeding or transplanting, unlike the MC (Flinn et al., 1988; Oad et al., 2002); thus, ratooning is the most suitable cropping system in areas where labor and water are in short supply. Due to its shorter cropping duration, fewer inputs, such as fertilizer and pesticides, are needed in RCs. For the merits mentioned above, ratooning is practiced increasingly in the USA, Southeast Asia and China (Nakano et al., 2007).

Studies have shown that attention should be given to achieving ratooning success. Variety characteristics are the major factor for ratoon success because varieties used in ratooning will be exposed to different day lengths, 
temperatures and amounts of sunlight in the main season and ratoon season, so varieties with wider adaptability behave better in ratooning practice (Sun et al., 1988).

Low temperatures after the main season will prevent ratoon development, so earlier planting is another factor for ratoon success. In addition, it has been reported that the harvest mode of the main crop will also affect ratooning. First, the best harvest time for the main crop is before full maturity, while the culms are still greenish in color; late harvest will reduce the number of auxiliary buds sprouting and thus reduce productive tiller and ratoon yields (Ichii et al., 1981; Xiong et al., 1991). Second, the stubble is left with a 2-3 node or 20-40 cm height because panicles from the upper node contribute more to the ratoon yield than those from the lower node (Yi et al., 2009; He et al., 2014). Insect pests and disease control during the main crop also play key roles in ratooning. Unlike a rice-rice rotation system, ratooning prolonged the duration of rice serving as a host of diseases or insect pests, so these biotic stresses in the main crop would affect ratoon tiller production, thereby reducing ratoon yield. Therefore, varieties with high biotic stress resistance are very important for ratooning (Dela Cruz et al., 1988).

The grain quality of rice, including grain appearance, grain size, and grain milling quality, is genetically controlled by quantitative trait loci (QTLs) or genes such as Chalky 5, qPGWC-7 and UGPase1, which control grain chalkiness (Woo et al., 2008; Zhou et al., 2009; Li et al., 2014); $W x$ and DULL, which affect grain translucency (Wan et al., 2007; Zeng et al., 2007); and GW2, GS3 and GW5, which control grain size (Mao et al., 2010; Liu et al., 2017; Choi et al., 2018). Some QTLs control grain size also have an effect on grain chalkiness, such as upregulation of $G W 7$ expression is correlated with more slender grains and less chalky grains (Wang et al., 2015a); overexpression of GL7 resulted in an increase in grain length and improve in grain chalkiness (Wang et al., 2015b). Head rice recovery is influenced by grain characteristics, such as chalkiness, grain shape, and grain moisture (Moldenhauer et al., 2004), and some QTLs related to milled rice recovery and head rice recovery have been mapped (Mei et al., 2002; Septiningsih et al., 2003).

Environmental conditions, especially temperature, also have a great influence on grain quality. It has been reported that high nighttime temperatures during the filling stage increase grain chalkiness (Cooper et al., 2008; Ishimaru et al., 2009) and decrease grain weight and grain translucency (Peng et al., 2004; Ishimaru et al., 2009). As mentioned above, the main crop and ratoon crop are genetically the same, but they are exposed to different environments during growth and development, so different grain quality could be expected between them. However, no detailed study has been conducted yet. In addition, variety identification is practiced in the United States, and the harvests from the MC are always mixed with the RC in a bin of the same variety to facilitate storage. However, it is not clear whether the quality difference in the MC and the RC affects the practice of identity preservation. Therefore, a detailed study comparing grain quality between the MC and the RC will be a helpful guide in ratoon rice production.

In this study, a two-year field trial was conducted using 18 elite tropical Japonica breeding lines and two check (CK) varieties to identify the grain quality differences between the MC and the RC, determine the potential effects of mixing MC and RC harvests and screen for elite breeding lines with high total yield for ratooning rice production.

\section{Method}

\subsection{Rice Entries and Culture}

The two-year field experiments were conducted at Eagle Lake ( $\left.29^{\circ} 37^{\prime} \mathrm{N}, 96^{\circ} 22^{\prime} \mathrm{W}\right)$, Texas, USA. Each year, 18 elite tropical Japonica breeding lines with similar growth durations and two CK varieties, ANTONIO as a yield check and PRESIDIO as a grain quality check for $\mathrm{MC} / \mathrm{RC}$, were planted in plots arranged in a completely randomized block design with three replications. Each plot had 6-meter rows spaced $25 \mathrm{~cm}$ apart. Entries were directly seeded at a rate of $90 \mathrm{~kg} \mathrm{ha}^{-1}$. Fertilizer for the MC was applied in a 3-way split: preplanting at 50-50-50 $\mathrm{kg}$ NPK ha ${ }^{-1}$ using Triple 13, preflooding at $100 \mathrm{~kg} \mathrm{~N} \mathrm{ha}^{-1}$ using urea and at panicle initiation at $90 \mathrm{~kg} \mathrm{~N} \mathrm{ha}^{-1}$ using ammonium sulfate. A fertilizer rate of $90 \mathrm{~kg} \mathrm{~N}^{-1}$ using urea was applied immediately after the MC harvest as ratoon fertilizer. The Texas rice production guidelines were followed for the other cultural practices. A rice combine harvester was used in both the $\mathrm{MC}$ and $\mathrm{RC}$ harvests with cutting heights of approximately 38 and $25 \mathrm{~cm}$, respectively. 
Table 1. Comparison of main crop (MC) and ratoon crop (RC) mean chalkiness and translucency in tested breeding lines and two varieties

\begin{tabular}{|c|c|c|c|c|c|c|c|c|c|}
\hline \multirow{2}{*}{ No. } & \multirow{2}{*}{ Breeding Line/Variety } & \multicolumn{4}{|c|}{ Chalkiness } & \multicolumn{4}{|c|}{ White Vitreous } \\
\hline & & $2016 \mathrm{MC}$ & $2016 \mathrm{RC}$ & $2017 \mathrm{MC}$ & $2017 \mathrm{RC}$ & $2016 \mathrm{MC}$ & $2016 \mathrm{RC}$ & $2017 \mathrm{MC}$ & $2017 \mathrm{RC}$ \\
\hline 1 & ANTONIO & $11.37 \mathrm{a}$ & $3.17 \mathrm{~b}$ & $9.48 \mathrm{a}$ & $1.52 \mathrm{~b}$ & $129.26 \mathrm{a}$ & $113.27 \mathrm{a}$ & $127.31 \mathrm{a}$ & $127.23 \mathrm{a}$ \\
\hline 2 & RU0803147 & $14.70 \mathrm{a}$ & $1.11 \mathrm{c}$ & $8.86 \mathrm{~b}$ & $0.95 \mathrm{c}$ & $129.15 \mathrm{a}$ & $127.23 \mathrm{a}$ & $128.93 \mathrm{a}$ & $126.55 \mathrm{a}$ \\
\hline 3 & RU0803153 & $12.06 \mathrm{a}$ & $1.43 \mathrm{c}$ & $8.29 \mathrm{~b}$ & $1.29 \mathrm{c}$ & $129.71 \mathrm{a}$ & $128.58 \mathrm{a}$ & $129.33 \mathrm{a}$ & $126.70 \mathrm{~b}$ \\
\hline 4 & RU1303138 & $6.96 \mathrm{a}$ & $1.77 \mathrm{~b}$ & $6.43 \mathrm{a}$ & $1.24 \mathrm{~b}$ & $128.57 \mathrm{a}$ & $128.63 \mathrm{a}$ & $127.88 \mathrm{a}$ & $126.27 \mathrm{~b}$ \\
\hline 5 & RU1303153 & $7.79 \mathrm{a}$ & $1.92 \mathrm{~b}$ & $7.87 \mathrm{a}$ & $1.20 \mathrm{~b}$ & $128.72 \mathrm{a}$ & $128.59 \mathrm{a}$ & $128.36 \mathrm{a}$ & $126.85 \mathrm{~b}$ \\
\hline 6 & RU1303181 & $5.07 \mathrm{a}$ & $1.78 \mathrm{~b}$ & $3.52 \mathrm{a}$ & $1.43 \mathrm{~b}$ & $129.19 \mathrm{a}$ & $128.50 \mathrm{a}$ & $127.44 \mathrm{a}$ & $127.18 \mathrm{a}$ \\
\hline 7 & RU1403138 & $4.23 \mathrm{~b}$ & $1.58 \mathrm{~b}$ & $7.27 \mathrm{a}$ & $2.38 \mathrm{~b}$ & $129.78 \mathrm{a}$ & $129.25 \mathrm{a}$ & $128.99 \mathrm{ab}$ & $128.29 \mathrm{~b}$ \\
\hline 8 & RU1403141 & $3.81 \mathrm{a}$ & $0.81 \mathrm{c}$ & $2.70 \mathrm{~b}$ & $0.99 \mathrm{c}$ & $129.82 \mathrm{a}$ & $128.10 \mathrm{a}$ & $127.27 \mathrm{~b}$ & $126.02 \mathrm{~b}$ \\
\hline 9 & RU1503147 & $5.64 \mathrm{a}$ & $0.66 \mathrm{~b}$ & $5.12 \mathrm{a}$ & $1.21 \mathrm{~b}$ & $129.57 \mathrm{a}$ & $127.06 \mathrm{a}$ & $127.12 \mathrm{~b}$ & $126.20 \mathrm{~b}$ \\
\hline 10 & RU1503169 & $14.21 \mathrm{a}$ & $1.90 \mathrm{c}$ & $7.77 \mathrm{~b}$ & $0.70 \mathrm{c}$ & $128.70 \mathrm{a}$ & $128.01 \mathrm{a}$ & $128.82 \mathrm{a}$ & $125.83 \mathrm{~b}$ \\
\hline 11 & RU1503175 & $15.01 \mathrm{a}$ & $0.91 \mathrm{c}$ & $10.96 \mathrm{~b}$ & $1.02 \mathrm{c}$ & $128.37 \mathrm{a}$ & $127.58 \mathrm{a}$ & $128.34 \mathrm{a}$ & $126.39 \mathrm{a}$ \\
\hline 12 & RU1603086 & $4.11 \mathrm{a}$ & $2.20 \mathrm{ab}$ & $3.87 \mathrm{a}$ & $1.19 \mathrm{~b}$ & $129.72 \mathrm{a}$ & $128.90 \mathrm{a}$ & $128.50 \mathrm{a}$ & $128.04 \mathrm{a}$ \\
\hline 13 & RU1603089 & $10.29 \mathrm{a}$ & $1.51 \mathrm{c}$ & $5.79 \mathrm{~b}$ & $1.26 \mathrm{c}$ & $129.00 \mathrm{a}$ & $128.66 \mathrm{a}$ & $127.97 \mathrm{a}$ & $128.08 \mathrm{a}$ \\
\hline 14 & RU1603113 & $3.48 \mathrm{a}$ & $1.51 \mathrm{a}$ & $3.40 \mathrm{a}$ & $1.44 \mathrm{a}$ & $129.01 \mathrm{a}$ & $128.42 \mathrm{a}$ & $128.21 \mathrm{a}$ & $127.53 \mathrm{a}$ \\
\hline 15 & RU1603116 & $2.76 \mathrm{a}$ & $1.24 \mathrm{~b}$ & $1.90 \mathrm{ab}$ & $1.49 \mathrm{ab}$ & $130.54 \mathrm{a}$ & $128.40 \mathrm{a}$ & $128.29 \mathrm{~b}$ & $128.15 \mathrm{~b}$ \\
\hline 16 & RU1603138 & $9.18 \mathrm{a}$ & $1.15 \mathrm{~b}$ & $4.81 \mathrm{~b}$ & $1.27 \mathrm{~b}$ & $130.70 \mathrm{a}$ & $128.48 \mathrm{a}$ & $128.58 \mathrm{ab}$ & $127.48 \mathrm{~b}$ \\
\hline 17 & RU1603166 & $3.68 \mathrm{a}$ & $2.70 \mathrm{a}$ & $3.34 \mathrm{a}$ & $1.72 \mathrm{a}$ & $129.18 \mathrm{a}$ & $128.92 \mathrm{a}$ & $126.06 \mathrm{~b}$ & $126.96 \mathrm{ab}$ \\
\hline 18 & RU1603178 & $5.00 \mathrm{a}$ & $3.28 \mathrm{a}$ & $3.84 \mathrm{a}$ & $1.71 \mathrm{a}$ & $131.31 \mathrm{a}$ & $129.62 \mathrm{a}$ & $129.48 \mathrm{a}$ & $129.11 \mathrm{a}$ \\
\hline 19 & RU1603187 & $4.33 \mathrm{a}$ & $1.82 \mathrm{~b}$ & $3.95 \mathrm{a}$ & $1.08 \mathrm{~b}$ & $129.18 \mathrm{a}$ & $128.50 \mathrm{a}$ & $126.43 \mathrm{a}$ & $127.09 \mathrm{a}$ \\
\hline 20 & PRESIDIO & $2.50 \mathrm{a}$ & $0.90 \mathrm{~b}$ & $2.92 \mathrm{a}$ & $1.51 \mathrm{~b}$ & $131.69 \mathrm{a}$ & $127.61 \mathrm{a}$ & $129.91 \mathrm{~b}$ & $127.84 \mathrm{c}$ \\
\hline Mean & & $7.31 \mathrm{a}$ & $1.67 \mathrm{c}$ & $5.60 \mathrm{~b}$ & $1.33 \mathrm{~d}$ & $129.56 \mathrm{a}$ & $128.37 \mathrm{~b}$ & $128.16 b$ & $127.19 \mathrm{c}$ \\
\hline
\end{tabular}

Note. Data followed by different letters within one row denote a significant difference between the main crop and ratoon crop in a given year (difference was calculated for four data points over two years) for each test entry at the 5\% level according to the LSD test. Means followed by bold letters within one row denote significant differences between the main crop and ratoon crop across two years for all test entries at the $5 \%$ level according to the LSD test.

\subsection{Data Recorded}

Four inner rows in a plot were harvested as they approached $20 \%$ grain moisture. Rough (unmilled) rice samples were dried to $12 \%$ moisture using an ambient-forced-air dryer. Rice milling was performed using a PAZ 1 Zaccaria mill (Zaccaria, USA), while an S21 Rice Statistical Analyzer (TKD Tecnologia, Brazil) was used to evaluate milled rice for chalkiness, translucency and grain size (length and width). Chalky grains were defined as grains with chalky areas of at least $50 \%$.

\subsection{Data Analysis}

Data were analyzed using SPSS 22 (IBM Corp). The difference between the MC and the RC was determined using the least significance difference (LSD) test at the 0.05 probability level. Graphical representation of the data was made using Excel and CorelDRAW X8. 


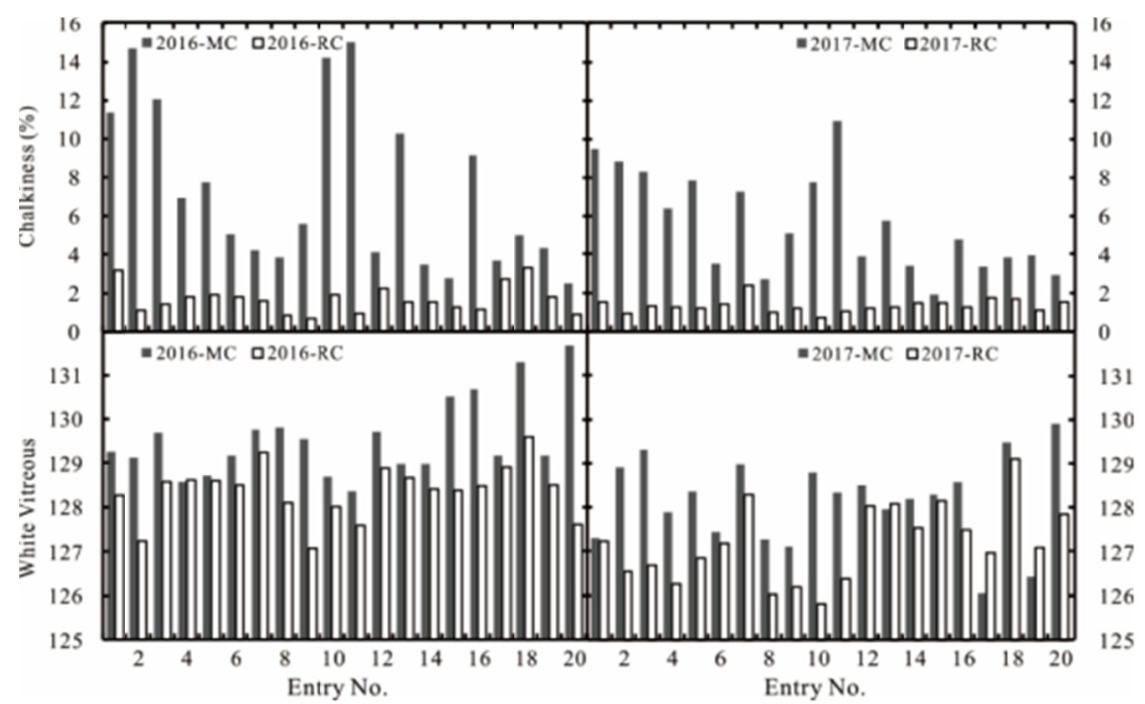

Figure 1. Yearly variation in main crop (MC) and ratoon crop (RC) chalkiness and translucency of tested breeding lines and two varieties

\section{Results}

\subsection{Chalkiness and Translucency Performance in MC and RC Across Two Years}

Across the two years, the MC chalkiness ranged from 1.90 to $15.01 \%$, with a mean value of $6.46 \%$, and the RC ranged from 0.66 to $3.28 \%$, with a mean value of $1.50 \%$. On average, the RC had significantly lower chalkiness than the MC for nearly all tested lines, and the average RC chalkiness was $5.64 \%$ and $4.27 \%$ lower than that in the MC in 2016 and 2017, respectively. In addition, the mean yearly chalkiness was higher in 2016 than in 2017 for both the MC and the RC (Table 1; Figure 1).

Table 2. F-values for three-way analysis of variance (ANOVA) for different traits

\begin{tabular}{llllllll}
\hline Source & Chalkiness & Translucency & Grain Length & Grain Width & Milled Rice Recovery & Head Rice Recovery & Yield \\
\hline Year (Y) & $43.87^{* *}$ & $5.73^{*}$ & $200.93^{* *}$ & $56.02^{* *}$ & $76.01^{* *}$ & $45.12^{* *}$ & 3.72 \\
Crop Type (CT) & $1035.92^{* *}$ & $14.63^{* *}$ & 2.41 & $4.54^{*}$ & $31.17^{* *}$ & 3.74 & $2173.68^{* *}$ \\
Entry (E) & $22.25^{* *}$ & 1.67 & $24.46^{* *}$ & $49.60^{* *}$ & $2.07^{* *}$ & $1.78^{*}$ & $9.43^{* *}$ \\
Y $\times$ CT & $19.74^{* *}$ & 1.62 & $44.16^{* *}$ & $29.86^{* *}$ & 0.09 & 0.02 & $16.61^{* *}$ \\
Y $\times$ E & $3.59^{* *}$ & 1.02 & $3.12^{* *}$ & 1.56 & 1.23 & $2.57^{* *}$ & $1.85^{*}$ \\
CT $\times$ E & $24.52^{* *}$ & 1.05 & $4.02^{* *}$ & $12.23^{* *}$ & 0.62 & 1.48 & $3.35^{* *}$ \\
Y $\times$ CT $\times$ E & $2.87^{* *}$ & 1.2 & $2.57^{* *}$ & $2.29^{* *}$ & 0.52 & 0.75 & 0.47 \\
\hline
\end{tabular}

Note. ${ }^{*}: \mathrm{P}<0.05 ;{ }^{* *}: \mathrm{P}<0.01$.

White vitreous (WV) readings are indicative of grain translucency. Higher values suggest lower (inferior) translucency. The majority of test entries showed that RC entries had lower WV (higher translucency) than the MC entries in each year, but only $25 \%$ of entries in 2016 or 2017 showed significant differences between MC and RC. The mean yearly WV in MC and RC in 2016 was significantly higher than that in 2017 (Table 1; Figure $1)$.

A three-way analysis of variance indicated that, for chalkiness, Year (Y), Crop Type (CT, MC or RC) and Entry (E) individually and in 2 or 3-way interactions affected chalkiness significantly, but CT had the most significant effect on chalkiness. For grain translucency, only CT and Y had significant effects on grain translucency (Table 2). 
Table 3. Comparison of mean milled grain length and width in main crop (MC) and ratoon crop (RC) of tested breeding lines and two varieties

\begin{tabular}{|c|c|c|c|c|c|c|c|c|c|}
\hline \multirow{2}{*}{ No. } & \multirow{2}{*}{ Breeding Line/Variety } & \multicolumn{4}{|c|}{ Grain length } & \multicolumn{4}{|c|}{ Grain width } \\
\hline & & $2016 \mathrm{MC}$ & 2016 RC & $2017 \mathrm{MC}$ & $2017 \mathrm{RC}$ & $2016 \mathrm{MC}$ & $2016 \mathrm{RC}$ & $2017 \mathrm{MC}$ & $2017 \mathrm{RC}$ \\
\hline 1 & ANTONIO & $6.37 \mathrm{a}$ & $6.47 \mathrm{a}$ & $6.42 \mathrm{a}$ & $6.27 \mathrm{a}$ & $1.84 \mathrm{a}$ & $1.94 \mathrm{a}$ & $1.89 \mathrm{a}$ & $1.88 \mathrm{a}$ \\
\hline 2 & RU0803147 & $6.49 \mathrm{a}$ & $6.45 \mathrm{a}$ & $6.39 \mathrm{a}$ & $6.24 \mathrm{~b}$ & $1.92 \mathrm{ab}$ & $1.87 \mathrm{~b}$ & $1.98 \mathrm{a}$ & $1.90 \mathrm{~b}$ \\
\hline 3 & RU0803153 & $6.49 \mathrm{a}$ & $6.43 \mathrm{a}$ & $6.39 \mathrm{a}$ & $6.35 \mathrm{a}$ & $1.93 \mathrm{ab}$ & $1.92 \mathrm{ab}$ & $1.99 \mathrm{a}$ & $1.90 \mathrm{~b}$ \\
\hline 4 & RU1303138 & $6.34 \mathrm{~b}$ & $6.42 \mathrm{ab}$ & $6.47 \mathrm{a}$ & $6.34 \mathrm{~b}$ & $1.91 \mathrm{a}$ & $1.89 \mathrm{a}$ & $1.97 \mathrm{a}$ & $1.92 \mathrm{a}$ \\
\hline 5 & RU1303153 & $6.24 \mathrm{a}$ & $6.40 \mathrm{a}$ & $6.37 \mathrm{a}$ & $6.28 \mathrm{a}$ & $1.90 \mathrm{~b}$ & $1.91 \mathrm{~b}$ & $1.97 \mathrm{a}$ & $1.89 \mathrm{~b}$ \\
\hline 6 & RU1303181 & $6.70 \mathrm{a}$ & $6.59 \mathrm{a}$ & $6.59 \mathrm{a}$ & $6.42 \mathrm{~b}$ & $1.9 \mathrm{ab}$ & $1.88 \mathrm{~b}$ & $1.95 \mathrm{a}$ & $1.92 \mathrm{ab}$ \\
\hline 7 & RU1403138 & $6.51 \mathrm{a}$ & $6.45 \mathrm{a}$ & $6.28 \mathrm{a}$ & $6.25 \mathrm{a}$ & $2.04 \mathrm{~b}$ & $1.95 \mathrm{c}$ & $2.10 \mathrm{a}$ & $1.94 \mathrm{c}$ \\
\hline 8 & RU1403141 & $6.43 \mathrm{ab}$ & $6.58 \mathrm{a}$ & $6.13 \mathrm{~b}$ & $6.24 \mathrm{~b}$ & $1.85 \mathrm{a}$ & $1.86 \mathrm{a}$ & $1.89 \mathrm{a}$ & $1.89 \mathrm{a}$ \\
\hline 9 & RU1503147 & $6.63 \mathrm{a}$ & $6.67 \mathrm{a}$ & $6.54 \mathrm{~b}$ & $6.33 \mathrm{c}$ & $1.91 \mathrm{a}$ & $1.93 \mathrm{a}$ & $1.94 \mathrm{a}$ & $1.91 \mathrm{a}$ \\
\hline 10 & RU1503169 & $6.44 \mathrm{ab}$ & $6.51 \mathrm{a}$ & $6.35 \mathrm{~b}$ & $6.20 \mathrm{c}$ & $1.89 \mathrm{a}$ & $1.88 \mathrm{a}$ & $1.95 \mathrm{a}$ & $1.84 \mathrm{a}$ \\
\hline 11 & RU1503175 & $6.44 \mathrm{a}$ & $6.48 \mathrm{a}$ & $6.33 \mathrm{a}$ & $6.29 \mathrm{a}$ & $1.89 \mathrm{~b}$ & $1.89 \mathrm{~b}$ & $1.96 \mathrm{a}$ & $1.90 \mathrm{~b}$ \\
\hline 12 & RU1603086 & $6.5 \mathrm{ab}$ & $6.55 \mathrm{a}$ & $6.27 \mathrm{~b}$ & $6.40 \mathrm{ab}$ & $1.83 \mathrm{~b}$ & $1.86 \mathrm{~b}$ & $1.86 \mathrm{~b}$ & $1.90 \mathrm{a}$ \\
\hline 13 & RU1603089 & $6.56 \mathrm{a}$ & $6.59 \mathrm{a}$ & $6.64 \mathrm{a}$ & $6.31 \mathrm{a}$ & $1.87 \mathrm{~b}$ & $1.87 \mathrm{~b}$ & $1.95 \mathrm{a}$ & $1.87 \mathrm{~b}$ \\
\hline 14 & RU1603113 & $6.59 \mathrm{a}$ & $6.44 \mathrm{a}$ & $6.36 \mathrm{a}$ & $6.29 \mathrm{a}$ & $1.91 \mathrm{ab}$ & $1.89 \mathrm{~b}$ & $1.97 \mathrm{a}$ & $1.95 \mathrm{ab}$ \\
\hline 15 & RU1603116 & $6.86 \mathrm{a}$ & $6.90 \mathrm{a}$ & $6.94 \mathrm{a}$ & $6.53 \mathrm{~b}$ & $1.78 \mathrm{bc}$ & $1.80 \mathrm{~b}$ & $1.77 \mathrm{c}$ & $1.84 \mathrm{a}$ \\
\hline 16 & RU1603138 & $6.61 \mathrm{~b}$ & $6.77 \mathrm{a}$ & $6.46 \mathrm{bc}$ & $6.38 \mathrm{c}$ & $1.89 \mathrm{a}$ & $1.86 \mathrm{a}$ & $1.91 \mathrm{a}$ & $1.90 \mathrm{a}$ \\
\hline 17 & RU1603166 & $6.39 \mathrm{~b}$ & $6.48 \mathrm{a}$ & $6.34 \mathrm{~b}$ & $6.35 \mathrm{~b}$ & $1.75 \mathrm{~b}$ & $1.85 \mathrm{a}$ & $1.74 \mathrm{~b}$ & $1.83 \mathrm{a}$ \\
\hline 18 & RU1603178 & $6.34 \mathrm{a}$ & $6.45 \mathrm{a}$ & $6.16 \mathrm{a}$ & $6.28 \mathrm{a}$ & $1.86 \mathrm{~b}$ & $1.89 \mathrm{ab}$ & $1.90 \mathrm{a}$ & $1.88 \mathrm{ab}$ \\
\hline 19 & RU1603187 & $6.41 \mathrm{a}$ & $6.49 \mathrm{a}$ & $6.28 \mathrm{a}$ & $6.24 \mathrm{a}$ & $1.74 \mathrm{~b}$ & $1.80 \mathrm{ab}$ & $1.75 \mathrm{~b}$ & $1.84 \mathrm{a}$ \\
\hline 20 & PRESIDIO & $\begin{array}{r}6.27 \mathrm{a} \\
-\end{array}$ & $\begin{array}{r}6.56 \mathrm{a} \\
-\end{array}$ & $6.32 \mathrm{a}$ & $6.30 \mathrm{a}$ & $1.79 \mathrm{~b}$ & $1.89 \mathrm{a}$ & $1.87 \mathrm{a}$ & $1.88 \mathrm{a}$ \\
\hline Mean & & $6.48 \mathrm{~b}$ & $6.53 \mathrm{a}$ & $6.40 \mathrm{c}$ & $6.32 \mathrm{~d}$ & $1.87 \mathrm{~b}$ & $1.88 \mathrm{~b}$ & $1.91 \mathrm{a}$ & $1.89 \mathrm{ab}$ \\
\hline
\end{tabular}

Note. Data followed by different letters within one row denote significant differences between the main crop and ratoon crop in a given year for each test entry at the 5\% level according to the LSD test. Means followed by bold letters within one row denote significant differences between the main crop and ratoon crop across two years for all test entries at the $5 \%$ level according to the LSD test.

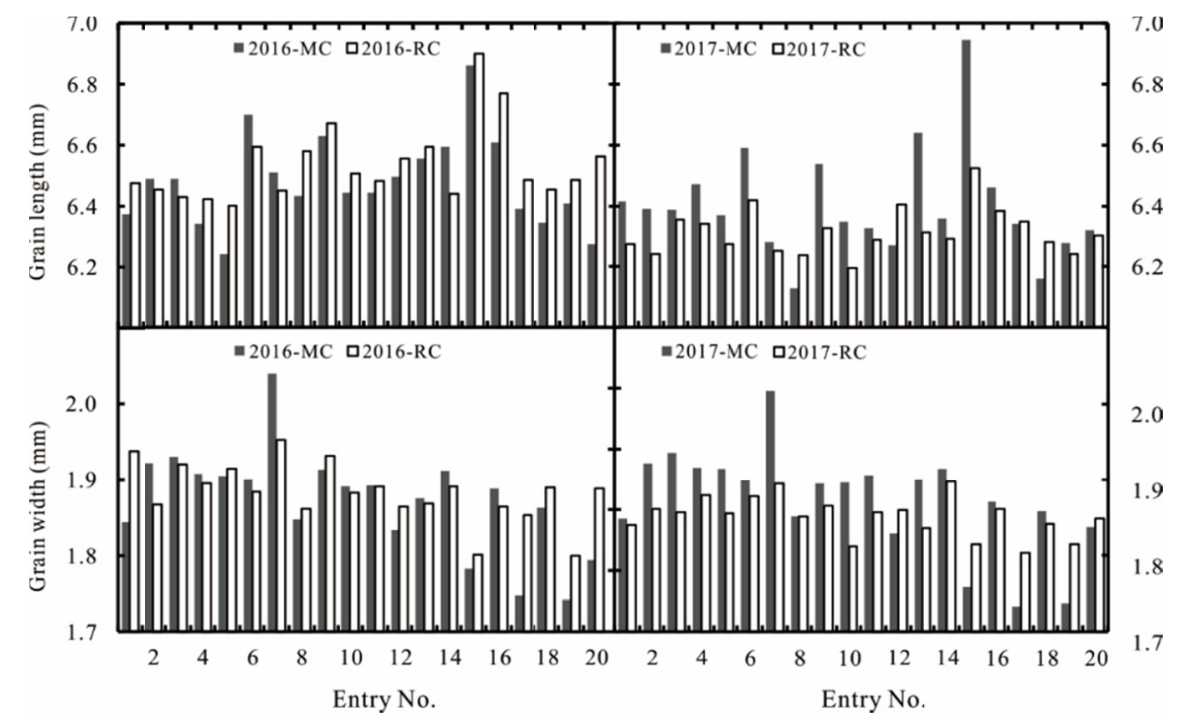

Figure 2. Yearly variation in grain length and width in main crop (MC) and ratoon crop (RC) of tested breeding lines and varieties

\subsection{Grain Size Performance in the MC and RC Across Two Years}

The mean grain length was significantly longer in 2016 than in 2017 for both the MC and the RC. RC grains were significantly longer than MC grains on average in 2016, but the reverse was true in 2017. The variation in grain width was relatively small, and no difference was observed between the $\mathrm{MC}$ and $\mathrm{RC}$ within the same year; only the MC in 2017 was wider than the MC and RC in 2016 (Table 3; Figure 2). 
A three-way analysis of variance indicated that the Year effect was most significant on both grain length and width; in addition, they were affected by $\mathrm{E}, \mathrm{Y} \times \mathrm{CT}, \mathrm{CT} \times \mathrm{E}$ and $\mathrm{Y} \times \mathrm{CT} \times \mathrm{E}($ Table 2$)$.

Milled rice recovery and head rice recovery performance in the $\mathrm{MC}$ and $\mathrm{RC}$.

Table 4. Comparison of mean milled rice recovery and head rice recovery in main crop (MC) and ratoon crop (RC) of tested breeding lines and two varieties

\begin{tabular}{|c|c|c|c|c|c|c|c|c|c|}
\hline \multirow{2}{*}{ No. } & \multirow{2}{*}{ Breeding Line/Variety } & \multicolumn{4}{|c|}{ Milled Rice Recovery } & \multicolumn{4}{|c|}{ Head Rice Recovery } \\
\hline & & $2016 \mathrm{MC}$ & $2016 \mathrm{RC}$ & $2017 \mathrm{MC}$ & $2017 \mathrm{RC}$ & $2016 \mathrm{MC}$ & $2016 \mathrm{RC}$ & $2017 \mathrm{MC}$ & $2017 \mathrm{RC}$ \\
\hline 1 & ANTONIO & $75.06 \mathrm{a}$ & $73.29 \mathrm{a}$ & $72.46 \mathrm{a}$ & $72.13 \mathrm{a}$ & $67.67 \mathrm{a}$ & $65.75 \mathrm{a}$ & $63.52 \mathrm{a}$ & $66.26 \mathrm{a}$ \\
\hline 2 & RU0803147 & $73.95 \mathrm{a}$ & $72.05 \mathrm{a}$ & $73.92 \mathrm{a}$ & $72.27 \mathrm{a}$ & $66.08 \mathrm{a}$ & $64.04 \mathrm{a}$ & $65.06 \mathrm{a}$ & $66.85 \mathrm{a}$ \\
\hline 3 & RU0803153 & $73.89 \mathrm{a}$ & $72.30 \mathrm{a}$ & $73.61 \mathrm{a}$ & $72.41 \mathrm{a}$ & $66.31 \mathrm{a}$ & $65.71 \mathrm{a}$ & $65.10 \mathrm{a}$ & $66.12 \mathrm{a}$ \\
\hline 4 & RU1303138 & $73.03 \mathrm{a}$ & $71.58 \mathrm{a}$ & $70.79 \mathrm{a}$ & $70.96 \mathrm{a}$ & $61.05 \mathrm{a}$ & $65.12 \mathrm{a}$ & $62.03 \mathrm{a}$ & $65.44 \mathrm{a}$ \\
\hline 5 & RU1303153 & $72.89 \mathrm{a}$ & $71.74 \mathrm{a}$ & $70.39 \mathrm{a}$ & $70.87 \mathrm{a}$ & $61.10 \mathrm{a}$ & $65.95 \mathrm{a}$ & $59.54 \mathrm{a}$ & $65.80 \mathrm{a}$ \\
\hline 6 & RU1303181 & $73.81 \mathrm{a}$ & $73.17 \mathrm{a}$ & $72.08 \mathrm{ab}$ & $70.17 \mathrm{~b}$ & $68.15 \mathrm{a}$ & $67.14 \mathrm{ab}$ & $64.27 \mathrm{bc}$ & $62.56 \mathrm{c}$ \\
\hline 7 & RU1403138 & $75.18 \mathrm{a}$ & $73.50 \mathrm{ab}$ & $71.91 \mathrm{ab}$ & $70.25 \mathrm{~b}$ & $70.20 \mathrm{a}$ & $67.86 \mathrm{a}$ & $60.58 \mathrm{a}$ & $60.54 \mathrm{a}$ \\
\hline 8 & RU1403141 & $75.08 \mathrm{a}$ & $73.36 \mathrm{a}$ & $73.75 \mathrm{a}$ & $70.52 \mathrm{~b}$ & $69.08 \mathrm{a}$ & $67.82 \mathrm{a}$ & $69.08 \mathrm{a}$ & $62.77 \mathrm{~b}$ \\
\hline 9 & RU1503147 & $74.39 \mathrm{a}$ & $73.31 \mathrm{a}$ & $73.37 \mathrm{a}$ & $71.36 \mathrm{~b}$ & $64.63 \mathrm{a}$ & $66.31 \mathrm{a}$ & $59.29 \mathrm{~b}$ & $64.40 \mathrm{a}$ \\
\hline 10 & RU1503169 & $74.79 \mathrm{a}$ & $72.54 \mathrm{ab}$ & $70.75 \mathrm{~b}$ & $72.15 \mathrm{~b}$ & $65.88 \mathrm{a}$ & $66.93 \mathrm{a}$ & $60.72 \mathrm{a}$ & $66.41 \mathrm{a}$ \\
\hline 11 & RU1503175 & $74.98 \mathrm{a}$ & $72.16 \mathrm{~b}$ & $74.12 \mathrm{ab}$ & $72.92 \mathrm{ab}$ & $65.50 \mathrm{a}$ & $64.81 \mathrm{a}$ & $65.32 \mathrm{a}$ & $67.21 \mathrm{a}$ \\
\hline 12 & RU1603086 & $74.01 \mathrm{a}$ & $73.17 \mathrm{a}$ & $71.81 \mathrm{a}$ & $71.47 \mathrm{a}$ & $67.79 \mathrm{a}$ & $67.67 \mathrm{a}$ & $63.77 \mathrm{a}$ & $64.05 \mathrm{a}$ \\
\hline 13 & RU1603089 & $74.31 \mathrm{a}$ & $73.99 \mathrm{a}$ & $72.96 \mathrm{a}$ & $71.55 \mathrm{a}$ & $61.66 \mathrm{a}$ & $68.63 \mathrm{a}$ & $63.68 \mathrm{a}$ & $64.41 \mathrm{a}$ \\
\hline 14 & RU1603113 & $74.31 \mathrm{a}$ & $73.42 \mathrm{ab}$ & $70.47 \mathrm{~b}$ & $70.43 \mathrm{~b}$ & $68.21 \mathrm{a}$ & $68.45 \mathrm{a}$ & $59.48 \mathrm{~b}$ & $62.47 \mathrm{ab}$ \\
\hline 15 & RU1603116 & $73.06 \mathrm{a}$ & $72.93 \mathrm{a}$ & $70.19 \mathrm{~b}$ & $70.20 \mathrm{~b}$ & $62.90 \mathrm{~b}$ & $67.31 \mathrm{a}$ & $59.76 \mathrm{c}$ & $60.56 \mathrm{bc}$ \\
\hline 16 & RU1603138 & $74.50 \mathrm{a}$ & $72.44 \mathrm{ab}$ & $73.18 \mathrm{a}$ & $70.06 \mathrm{~b}$ & $65.13 \mathrm{ab}$ & $65.89 \mathrm{ab}$ & $67.02 \mathrm{a}$ & $61.62 \mathrm{~b}$ \\
\hline 17 & RU1603166 & $74.75 \mathrm{a}$ & $73.30 \mathrm{a}$ & $73.11 \mathrm{a}$ & $69.17 \mathrm{~b}$ & $67.90 \mathrm{a}$ & $66.30 \mathrm{ab}$ & $62.01 \mathrm{ab}$ & $57.60 \mathrm{~b}$ \\
\hline 18 & RU1603178 & $72.71 \mathrm{a}$ & $72.28 \mathrm{a}$ & $69.45 \mathrm{~b}$ & $68.92 \mathrm{~b}$ & $66.37 \mathrm{a}$ & $65.49 \mathrm{ab}$ & $57.54 \mathrm{c}$ & $59.39 \mathrm{bc}$ \\
\hline 19 & RU1603187 & $75.84 \mathrm{a}$ & $74.24 \mathrm{ab}$ & $72.16 b c$ & $70.88 \mathrm{c}$ & $68.99 \mathrm{a}$ & $70.01 \mathrm{a}$ & $61.21 \mathrm{~b}$ & $62.93 \mathrm{~b}$ \\
\hline 20 & PRESIDIO & $73.09 \mathrm{a}$ & $72.70 \mathrm{a}$ & $73.01 \mathrm{a}$ & $71.24 \mathrm{a}$ & $60.11 \mathrm{a}$ & $63.54 \mathrm{a}$ & $63.24 \mathrm{a}$ & $63.88 \mathrm{a}$ \\
\hline Mean & & $74.18 \mathrm{a}$ & $72.87 \mathrm{~b}$ & $72.17 \mathrm{c}$ & $71.00 \mathrm{~d}$ & $65.73 \mathrm{a}$ & $66.54 \mathrm{a}$ & $62.61 \mathrm{~b}$ & $63.56 \mathrm{~b}$ \\
\hline
\end{tabular}

Note. Data followed by different letters within one row denote significant differences between the main crop and ratoon crop in a given year for each test entry at the 5\% level according to the LSD test. Means followed by bold letters within one row denote significant differences between the main crop and ratoon crop across two years for all test entries at the 5\% level according to the LSD test.

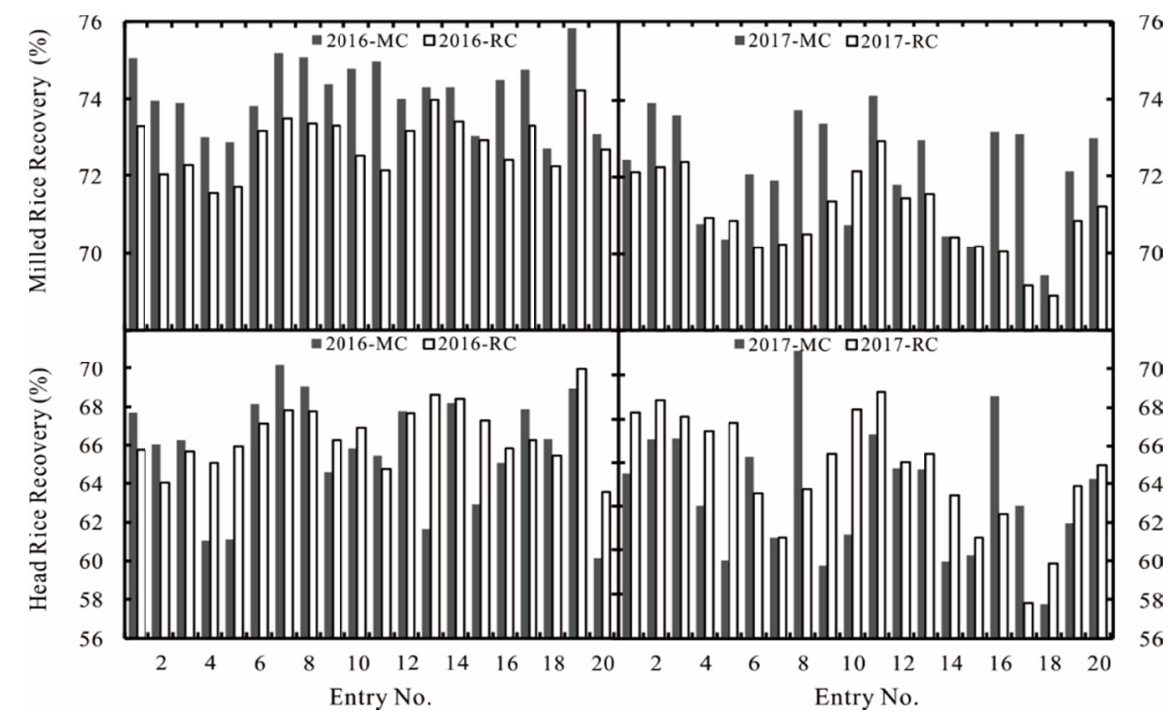

Figure 3. Yearly variation in milled rice recovery and head rice recovery in main crop (MC) and ratoon crop (RC) of tested breeding lines and varieties 


\subsection{Milled Rice Recovery and Head Rice Recovery Performance in the MC and RC Across Two Years}

The milled rice recovery for the MC was 1.8\% higher than that of the RC in 2016 and $1.65 \%$ higher than that of the $\mathrm{RC}$ in 2017. For head rice recovery, there was no significant difference between $\mathrm{MC}$ and RC within the same year, but both were higher in 2016 than in 2017, and head rice recovery was 4.83\% higher in 2016 than in 2017 on average for both MC and RC (Table 4; Figure 3). Year had the most significant effect on both milled rice recovery and head rice recovery. Crop Type and Entry also had significant effects on milled rice recovery, and Entry and $\mathrm{Y} \times \mathrm{E}$ had significant effects on head rice recovery (Table 2).

Table 5. Comparison of main crop (MC), ratoon crop (RC) and total $(\mathrm{MC}+\mathrm{RC})$ mean yield of tested breeding lines and two CK varieties

\begin{tabular}{|c|c|c|c|c|c|c|c|}
\hline \multirow{2}{*}{ No. } & \multirow{2}{*}{ Breeding Line/Variety } & \multicolumn{6}{|c|}{ Yield (t/ha) } \\
\hline & & $2016 \mathrm{MC}$ & $2016 \mathrm{RC}$ & 2016 Total $(\mathrm{MC}+\mathrm{RC})$ & $2017 \mathrm{MC}$ & $2017 \mathrm{RC}$ & 2017 Total $(\mathrm{MC}+\mathrm{RC})$ \\
\hline 1 & ANTONIO & $8.92 \mathrm{c}$ & $4.48 \mathrm{~d}$ & $13.41 \mathrm{a}$ & $10.14 \mathrm{~b}$ & $3.24 \mathrm{e}$ & $13.39 \mathrm{a}$ \\
\hline 2 & RU0803147 & $9.39 \mathrm{~b}$ & $3.69 \mathrm{c}$ & $13.08 \mathrm{a}$ & $9.77 \mathrm{~b}$ & $3.22 \mathrm{~d}$ & $12.99 \mathrm{a}$ \\
\hline 3 & RU0803153 & $9.37 \mathrm{~b}$ & $3.17 \mathrm{c}$ & $12.54 \mathrm{a}$ & $10.40 \mathrm{~b}$ & $3.08 \mathrm{c}$ & $13.48 \mathrm{a}$ \\
\hline 4 & RU1303138 & $8.49 \mathrm{~b}$ & $6.97 \mathrm{c}$ & $15.47 \mathrm{a}$ & $9.64 \mathrm{~b}$ & $5.59 \mathrm{c}$ & $15.24 \mathrm{a}$ \\
\hline 5 & RU1303153 & $7.39 \mathrm{~b}$ & $6.29 \mathrm{~d}$ & $13.68 \mathrm{a}$ & $9.02 \mathrm{c}$ & $5.37 \mathrm{~d}$ & $14.38 \mathrm{a}$ \\
\hline 6 & RU1303181 & $8.53 \mathrm{c}$ & $3.95 \mathrm{~d}$ & $12.48 \mathrm{a}$ & $10.31 \mathrm{~b}$ & $3.33 \mathrm{~d}$ & $13.64 \mathrm{a}$ \\
\hline 7 & RU1403138 & $8.24 \mathrm{c}$ & $3.64 \mathrm{~d}$ & $11.88 \mathrm{~b}$ & $11.20 \mathrm{~b}$ & $3.59 \mathrm{a}$ & $14.79 \mathrm{a}$ \\
\hline 8 & RU1403141 & $9.24 \mathrm{~b}$ & $4.54 \mathrm{c}$ & $13.78 \mathrm{a}$ & $9.43 \mathrm{~b}$ & $3.79 \mathrm{c}$ & $13.22 \mathrm{a}$ \\
\hline 9 & RU1503147 & $8.77 \mathrm{~b}$ & $4.94 \mathrm{c}$ & $13.71 \mathrm{a}$ & $9.90 \mathrm{~b}$ & $4.11 \mathrm{c}$ & $14.01 \mathrm{a}$ \\
\hline 10 & RU1503169 & $9.40 \mathrm{~b}$ & $3.16 \mathrm{c}$ & $12.55 \mathrm{a}$ & $8.92 \mathrm{~b}$ & $2.64 \mathrm{c}$ & $11.55 \mathrm{a}$ \\
\hline 11 & RU1503175 & $9.46 \mathrm{~b}$ & $3.25 \mathrm{c}$ & $12.71 \mathrm{a}$ & $9.62 \mathrm{~b}$ & $2.98 \mathrm{c}$ & $12.60 \mathrm{a}$ \\
\hline 12 & RU1603086 & $7.89 \mathrm{c}$ & $4.39 \mathrm{~d}$ & $12.28 \mathrm{a}$ & $9.72 \mathrm{~b}$ & $2.73 \mathrm{~d}$ & $12.45 \mathrm{a}$ \\
\hline 13 & RU1603089 & $8.92 \mathrm{~b}$ & $2.17 \mathrm{c}$ & $11.10 \mathrm{a}$ & $9.88 \mathrm{~b}$ & $2.59 \mathrm{c}$ & $12.48 \mathrm{a}$ \\
\hline 14 & RU1603113 & $8.51 \mathrm{~b}$ & $3.95 \mathrm{c}$ & $12.46 \mathrm{a}$ & $9.50 \mathrm{~b}$ & $3.35 \mathrm{c}$ & $12.85 \mathrm{a}$ \\
\hline 15 & RU1603116 & $9.52 \mathrm{c}$ & $5.19 \mathrm{~d}$ & $14.72 \mathrm{a}$ & $9.79 \mathrm{~b}$ & $4.35 \mathrm{~d}$ & $14.14 \mathrm{a}$ \\
\hline 16 & RU1603138 & $8.37 \mathrm{~b}$ & $3.34 \mathrm{c}$ & $11.71 \mathrm{a}$ & $9.72 \mathrm{~b}$ & $3.13 \mathrm{c}$ & $12.85 \mathrm{a}$ \\
\hline 17 & RU1603166 & $8.07 \mathrm{~b}$ & $2.67 \mathrm{c}$ & $9.86 \mathrm{a}$ & $8.18 \mathrm{~b}$ & $2.37 \mathrm{c}$ & $10.55 \mathrm{a}$ \\
\hline 18 & RU1603178 & $6.39 \mathrm{bc}$ & $5.48 \mathrm{c}$ & $11.87 \mathrm{a}$ & $8.62 \mathrm{~b}$ & $4.98 \mathrm{c}$ & $13.60 \mathrm{a}$ \\
\hline 19 & RU1603187 & $8.42 \mathrm{~b}$ & $3.39 \mathrm{c}$ & $10.68 \mathrm{a}$ & $8.11 \mathrm{~b}$ & $2.49 \mathrm{c}$ & $10.60 \mathrm{a}$ \\
\hline 20 & PRESIDIO & $7.91 \mathrm{~b}$ & $4.72 \mathrm{c}$ & $12.63 \mathrm{a}$ & $7.57 \mathrm{~b}$ & $3.70 \mathrm{c}$ & $11.26 \mathrm{a}$ \\
\hline Mean & & $8.56 \mathrm{c}$ & $4.07 \mathrm{~d}$ & $12.63 \mathrm{a}$ & $9.47 \mathrm{~b}$ & $3.53 \mathrm{e}$ & $13.00 \mathrm{a}$ \\
\hline
\end{tabular}

Note. Data followed by different letters within one row denote a significant difference in grain yield among the main crop, ratoon crop and total yield across two years for each entry at the 5\% level according to the LSD test. Means followed by bold letters within one row denote a significant difference in grain yield among the main crop, ratoon crop and total yield across two years for all test entries at the 5\% level according to the LSD test.

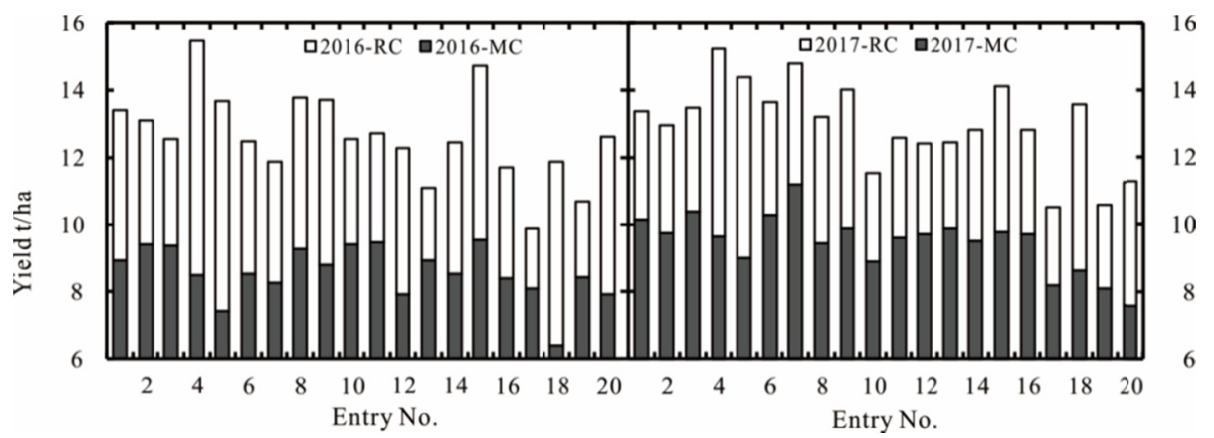

Figure 4. Yearly variation in main crop (MC) yield, ratoon crop (RC) yield and total yield of tested breeding lines and two varieties 


\subsection{Yield Performance in the MC and RC Across Two Years}

The MC in 2017 (with an average of $9.47 \mathrm{t} / \mathrm{ha}$ ) yielded 10.6\% more than the MC in 2016 (with an average of $8.56 \mathrm{t} / \mathrm{ha}$ ). However, the RC in 2017 (with an average of $3.53 \mathrm{t} / \mathrm{ha}$ ) yielded $13.30 \%$ lower than the RC in 2016 (with an average of $4.07 \mathrm{t} / \mathrm{ha}$ ); as a result, no significant difference was detected for total yield $(\mathrm{MC}+\mathrm{RC}$ ) between these two years, with values ranging from 9.86 to $15.47 \mathrm{t} / \mathrm{ha}$ and an average yield of 12.63 and 13.00 $\mathrm{t} / \mathrm{ha}$, respectively. The RC yield was approximately half of the MC yield in both years, with an average ratio of 47.5 and $37.3 \%$ (Table 5; Figure 4). The total yield in the two years was statistically similar, but it varied with entry and crop type. All two-way interactions were found to be significant, but three-way interactions were not significant (Table 2).

Table 6. Correlation coefficients of all evaluated traits in main crop and ratoon crop

\begin{tabular}{|c|c|c|c|c|c|c|c|c|c|c|c|c|c|}
\hline & $\begin{array}{l}\text { MC- } \\
\text { CY }\end{array}$ & $\begin{array}{l}\text { MC- } \\
\text { WV }\end{array}$ & $\begin{array}{l}\text { MC- } \\
\text { GL }\end{array}$ & $\begin{array}{l}\text { MC- } \\
\text { GW }\end{array}$ & $\begin{array}{l}\text { MC- } \\
\text { MRR }\end{array}$ & $\begin{array}{l}\text { MC- } \\
\text { HRR }\end{array}$ & $\begin{array}{l}\text { MC- } \\
\text { YD }\end{array}$ & $\begin{array}{l}\text { RC- } \\
\mathrm{CY}\end{array}$ & $\begin{array}{l}\text { RC- } \\
\text { WV }\end{array}$ & $\begin{array}{l}\text { RC- } \\
\text { GL }\end{array}$ & $\begin{array}{l}\text { RC- } \\
\text { GW }\end{array}$ & $\begin{array}{l}\text { RC- } \\
\text { MRR }\end{array}$ & $\begin{array}{l}\text { RC- } \\
\text { HRR }\end{array}$ \\
\hline MC-WV & 0 & & & & & & & & & & & & \\
\hline MC-GL & -0.05 & 0.12 & & & & & & & & & & & \\
\hline MC-GW & $0.32^{*}$ & -0.02 & -0.09 & & & & & & & & & & \\
\hline MC-MRR & $0.31^{*}$ & 0.25 & 0.12 & -0.17 & & & & & & & & & \\
\hline MC-HRR & 0.1 & 0.23 & 0.11 & -0.08 & $0.78^{* *}$ & & & & & & & & \\
\hline MC-YD & 0.24 & $-0.39^{*}$ & 0.2 & $0.48^{* *}$ & -0.18 & -0.14 & & & & & & & \\
\hline $\mathrm{RC}-\mathrm{CY}$ & -0.02 & 0.19 & -0.12 & -0.16 & 0.1 & 0.18 & $-0.35^{*}$ & & & & & & \\
\hline RC-WV & -0.11 & $0.55^{* *}$ & 0.16 & -0.22 & 0.22 & 0.23 & $-0.46^{* *}$ & $0.62^{* *}$ & & & & & \\
\hline RC-GL & -0.02 & $0.57^{* *}$ & $0.62^{* *}$ & $-0.39^{*}$ & $0.44^{* *}$ & 0.28 & -0.22 & 0.05 & $0.48^{* *}$ & & & & \\
\hline RC-GW & 0.18 & 0.01 & -0.22 & $0.73^{* *}$ & -0.07 & -0.01 & 0.22 & 0.1 & -0.05 & -0.28 & & & \\
\hline RC-MRR & 0.25 & $0.51^{* *}$ & 0.26 & -0.13 & $0.74^{* *}$ & $0.58^{* *}$ & -0.25 & 0.08 & 0.29 & $0.53^{* *}$ & -0.13 & & \\
\hline RC-HRR & 0.27 & $0.38^{*}$ & 0.23 & 0.03 & $0.58^{* *}$ & $0.52^{* *}$ & -0.18 & 0 & 0.18 & $0.37^{*}$ & -0.09 & $0.91^{* *}$ & \\
\hline RC-YD & -0.11 & 0.29 & 0.01 & 0.12 & -0.25 & -0.24 & -0.26 & 0.12 & 0.17 & 0.19 & 0.25 & -0.09 & 0.01 \\
\hline
\end{tabular}

Note. The numbers in the table indicate the correlation coefficient between different traits in the main crop and ratoon crop. MC, main crop; RC, ratoon crop; $\mathrm{CY}$, chalkiness; $\mathrm{WV}$, white vitreous; GL, grain length; GW, grain width; MRR, milled rice recovery; HRR, head rice recovery; YD, Yield. ${ }^{*}: P<0.05 ;{ }^{* *}: P<0.01$.

\subsection{Correlation Analysis of All Evaluated Traits in the Main Crop and Ratoon Crop}

The correlation coefficients for the relationships of all quality-related traits and yield in $\mathrm{MC}$ and $\mathrm{RC}$ are shown in Table 6. Grain appearance, including grain chalkiness and translucency, correlated with grain size, e.g., MC chalkiness was positively correlated with $\mathrm{MC}$ grain width $(0.32)$, and $\mathrm{RC}$ white vitreous was positively correlated with RC grain length (0.48). In addition, increased chalkiness in the RC resulted in increased white vitreous (0.62). Milled rice recovery and head rice recovery were positively correlated in the MC and RC, and grain length was correlated with milled rice recovery and head rice recovery in the RC.

Yield was also correlated with grain size or grain quality traits, e.g., increasing grain width in the MC was accompanied by increasing $\mathrm{MC}$ yield (0.48), and increasing $\mathrm{MC}$ yield resulted in decreasing $\mathrm{MC}$ white vitreous $(-0.4)$. In addition, MC yield was negatively correlated with chalkiness and white vitreous in the RC, with correlation coefficients of -0.35 and -0.46 , respectively, but no correlation was observed for RC yield.

\section{Discussion}

The ratoon rice system, as an energy-saving crop production method, has gradually become more common worldwide, but little is known about the grain quality difference between the $\mathrm{MC}$ and $\mathrm{RC}$ within the same rice varieties. Our results suggested that ratoon rice had a better appearance, with $4.96 \%$ lower chalkiness than main crop rice and higher translucency for most test entries in the two years. Additionally, some breeding lines, such as RU1603166 and RU1603178, had comparable quality traits with quality CK varieties, with consistently low chalkiness in both the MC and RC for two years. Although the milled rice recovery in the MC was higher than in the RC in both years, there was no difference in head rice recovery between the MC and RC in the same year.

Because of the shorter growth stage for the $\mathrm{RC}$, approximately half that of the $\mathrm{MC}$, the $\mathrm{RC}$ yield was approximately half that of the MC on average. However, some elite breeding lines had better ratooning ability, 
e.g., RU1303138 and RU1303153 had similar MC and RC yields and higher total yields than CK ANTONIO, so these lines could be candidate donor varieties for ratoon production.

Rice chalkiness not only affects grain appearance and milling properties but also cooking quality, so it is a key determinant of the commercial value of milled rice. It has been well documented that chalkiness is controlled by polygenes (Wan et al., 2007; Woo et al., 2008; Zhou et al., 2009; Li et al., 2014; Gong et al., 2017), resulting from loose packing of starch granules in the grain endosperm (Zhang et al., 2013; Lu et al., 2018). However, chalkiness has also been reported to be sensitive to the environment, especially high temperatures during the grain filling stage, which accelerate ripening and result in loose packing of the starch granules, thereby greatly increasing the proportion of chalky grains (Cooper et al., 2008; Ishimaru et al., 2009; Chen et al., 2013). Tashiro et al. (1991) reported that a temperature of over $26{ }^{\circ} \mathrm{C}$ during the filling stage would significantly enhance chalkiness, and Laenoi et al. (2017) reported that a variety with the chalkiest grain in the hot season had nearly no chalkiness in the cool season. Ratoon rice is a different cultivation system. In contrast to the main crop, the temperature during the $\mathrm{RC}$ total growth period decreased gradually. Considering the weather data during the growth period of the $\mathrm{MC}$ and $\mathrm{RC}$ in two years, a comparison was made of the average maximum and minimum temperatures of the $\mathrm{MC}$ and the $\mathrm{RC}$ from heading to maturity covering most of the test entries. The average maximum and minimum temperatures for the $\mathrm{MC}$ were $30^{\circ} \mathrm{C}$ and $16{ }^{\circ} \mathrm{C}$ in 2016 and $30{ }^{\circ} \mathrm{C}$ and $19{ }^{\circ} \mathrm{C}$ in 2017 . These values were much higher than the maximum and minimum temperatures for the $\mathrm{RC}$, of $12{ }^{\circ} \mathrm{C}$ and $0{ }^{\circ} \mathrm{C}$ in 2016 and $10{ }^{\circ} \mathrm{C}$ and $4{ }^{\circ} \mathrm{C}$ in 2017 . The low temperature for the $\mathrm{MC}$ was nearly the highest temperature for the $\mathrm{RC}$. The RC, therefore, was exposed to much lower maximum and minimum temperatures compared with the $\mathrm{MC}$ during the grain filling stage, which may be the main reason for the lower chalkiness of the RC.

A three-way ANOVA indicated that crop type had the most significant effect on chalkiness, so temperature difference may have played the key role in determining the chalkiness of the MC and RC milled grains. The yearly difference in average temperature for both the MC and the RC further affected the degree of chalkiness. As expected, genotype is a factor in grain chalkiness, which could be due to the QTLs that were reported. However, the interaction of genotype with crop type and year and the three-way interaction obtained in this study support the complexity of chalkiness as a trait to focus on in breeding programs. These complex interactions could be the reason for slow progress in breeding for low chalk in rice. The stability of low chalk across years and crop type is a very desirable trait, and achieving it could be challenging. It can be noted, however, that some entries, such as RU1603166 and RU1603178, were stable over the two years and had nearly the same chalkiness in both the $\mathrm{MC}$ and the $\mathrm{RC}$; thus, they could be potential donors to a breeding program for low chalk.

It was reported that endosperm translucency had the highest correlation with chalkiness (Li et al., 2003b; Hao et al., 2009), and its formation was also similar to chalkiness, in that endosperm with tightly packed starch granules and no air space is more translucent than endosperm with loosely packed starch granules and more air space ( $\mathrm{Lu}$ et al., 2018); thus, those genes that are related to starch synthesis and packaging affect grain translucency (Wan et al., 2007; Zeng et al., 2007). Our results suggested the same; RC chalkiness had the highest correlation with $\mathrm{RC}$ white vitreous with a correlation coefficient of 0.62 , but no correlation was observed between chalkiness and white vitreous in the MC. As in chalkiness, crop type had the most significant effect on translucency. Similarly, the year affected translucency but not the entry or genotype. No interactions were observed, indicating that this trait is less complex than chalkiness. Based on these results, it can be concluded that translucency was determined mainly by the environment.

Head rice recovery is a high-priority objective for breeders because the market value of head grain is twice that of broken grain. Many QTLs related to head rice recovery have been mapped, and some of them overlapped with QTLs that control grain length because long grains tend to break easily (Tan et al., 2001; Nelson et al., 2011). However, our results showed that head rice recovery in RC was positively correlated with grain length.

Recent studies have identified several key QTLs and genes controlling grain size (Mao et al., 2010; Liu et al., 2017; Choi et al., 2018), and the molecular mechanisms of grain size regulation are gradually becoming clearer (Li et al., 2018). Aside from genetic control, it has been reported that environment has an effect on grain size; Funaba et al. (2006) reported that low temperature augmented grain weight for the extended grain maturity period. Our results indicated, although with lower average temperature and longer growth duration from heading to maturity, that some RC entries had smaller grain size (grain length or width) than the MC in 2017, but not in 2016. This may be due to environmental differences in different years and the shorter total growth period for RC. A three-way ANOVA also indicated that year had the most significant effect on both grain length and width, and crop type, but not entry, also affected grain width. 
Grain size was also correlated with other quality traits and yield, and our results showed that wider grain width resulted in higher chalkiness in the MC but not in the RC. A similar result was also reported by Song et al. (2007), that the $G W 2$ allele for increasing grain width had a negative effect on grain chalkiness. In addition, the $\mathrm{RC}$ grain length had a positive correlation with white vitreous readings in $\mathrm{MC}$ and $\mathrm{RC}$, indicating that increasing grain length had a negative effect on grain translucency.

Rice yield is a complex trait determined by its three components: grain weight, the number of grains per panicle and the number of panicles; many QTLs for these traits have been mapped, cloned or functionally characterized, e.g., OSNPF7.2 and MOC1 control tiller number in rice (Li et al., 2003a; Wang et al., 2018); GS3 and GW2 control grain size or weight (Mao et al., 2010; Choi et al., 2018); and Gn1a and PROG1 control grain number (Ashikari et al., 2005; Jin et al., 2008). In addition, yield always had a positive correlation with growth duration (Ying et al., 1998), so the RC yield was only approximately half that of the MC because the total growth duration of the RC was only approximately $60 \%$ that of MC. However, with the aid of different component-of-yield-related genes or QTLs in each entry, those with better ratooning ability could be selected as candidate donors for ratoon production. For example, RU1303138 had the highest total grain yield, and its RC yield was 82.10 and $58.00 \%$ of MC in 2016 and 2017, respectively. In addition, several others also yielded much better than the yield CK, ANTONIO.

A three-way ANOVA indicated that yield was a complex trait affected by many factors. Crop type had the most significant effect on yield, followed by entry; in addition, two-way interactions among years, crop type and entries were also observed. Yield was also correlated with quality traits, such as higher yield resulting in lower white vitreous (better translucency) in MC and RC.

A common practice in the U.S. while ratooning is to combine both $\mathrm{MC}$ and $\mathrm{RC}$ harvests of a variety in a bin for convenient storage and to maintain the varietal identity. Based on our results, we concluded that ratoon grain could help improve the quality of the mixture because of its good appearance (lower chalkiness and higher translucency). However, the instability of the grain size between the $\mathrm{MC}$ and the RC should be considered to minimize the nonuniformity of the grain size of the mixture. Currently, in China, rice grain is classified into four types according to its origin: ratoon grain, late-season grain, middle-season grain and early-season grain. The price decreases from the first classification to the fourth. The price of the RC grain is nearly two times that of the $\mathrm{MC}$ grain because, generally speaking, the ratoon crop, due to the low temperatures during its grain filling stage, has the best grain quality. Thus, for higher profit, the MC and RC could be stored and sold separately, which would be a good choice for U.S. rice growers.

\section{Conclusion}

A comparison of milled grain quality and yield from the MC and RC was conducted in this study, it was shown that RC grains had better appearance quality, including lower chalkiness and higher translucency, than the MC grains; in addition, some MC entries behaved larger grain size in one or two years. Therefore, RC harvests can be mixed with their corresponding MC harvests to improved total grain quality of a variety when variety identification is being practiced. For better uniformity in grain size of the mixture of $\mathrm{MC}$ and $\mathrm{RC}$, data on grain length and width should be considered during this practice. Some breeding lines had better and near stable ratooning ability and yield more than CKs, so they could be donors in breeding for ratoon rice production.

\section{References}

Ashikari, M., Sakakibara, H., Lin, S. Y., Yamamoto, T., Takashi, T., Nishimura, A., ... Matsuoka, M. (2005). Cytokinin oxidase regulates rice grain production. Science, 309(5735), 741-745. https://doi.org/10.1126/ science. 1113373

Cassman, K. G., Dobermann, A., Walters, D. T., \& Yang, H. S. (2003). Meeting cereal demand while protecting natural resources and improving environmental quality. Ann. Rev. Environ. Resour., 28(1), 315-358. https://doi.org/10.1146/annurev.energy.28.040202.122858

Chen, C., Huang, J. L., Zhu, L. Y., Shah, F., Nie, L. X., Cui, K. H., \& Peng, S. B. (2013). Varietal difference in the response of rice chalkiness to temperature during ripening phase across different sowing dates. Field Crops Res., 151, 85-91. https://doi.org/10.1016/j.fcr.2013.07.016

Chen, Q., He, A. B., Wang, W. Q., Peng, S. B., Huang, J. L., Cui, K. H., \& Nie, L. X. (2018). Comparisons of regeneration rate and yields performance between inbred and hybrid rice cultivars in a direct seeding rice-ratoon rice system in central China. Field Crops Res., 223, 164-170. https://doi.org/10.1016/ j.fcr.2018.04.010 
Choi, B. S., Kim, Y. J., Markkandan, K., Koo, Y. J., Song, J. T., \& Seo, H. S. (2018). GW2 functions as an E3 ubiquitin ligase for rice expansin-like 1. Int. J. Mol. Sci., 19(7), 1904-1916. https://doi.org/10.3390/ ijms 19071904

Cooper, N. T. W., Siebenmorgen, T. J., \& Counce, P. A. (2008). Effects of nighttime temperature during kernel development on rice physicochemical properties. Cereal Chem., 85(3), 276-282. https://doi.org/10.1094/ cchem-85-3-0276

Dela Cruz, C. G., \& Litisinger, J. A. (1988). Insect pests and their natural enemies in ratoon rice (pp. 195-208). Rice Ratooning IRRI, Manila, Philippines.

Flinn, J. C., \& Mercado, M. D. (1988). Economic perspectives on rice ratooning (pp. 17-29). Rice Ratooning IRRI, Manila, Philippines.

Funaba, M., Ishibashi, Y., Hossain, M. A., Iwanami, K., \& Iwaya-Inoue, M. (2006). Influence of low/high temperature on water status in developing and maturing rice grain. Plant Prod. Sci., 9(4), $347-354$. https://doi.org/10.1626/pps.9.347

Gong, J. Y., Miao, J. H., Zhao, Y., Zhao, Q., Feng, Q., Zhan, Q. L., ... Han, B. (2017). Dissecting genetic basis of grain shape and chalkiness traits in hybrid rice using multiple collaborative populations. Mol. Plant., 10(10), 1353-1356. https://doi.org/10.1016/j.molp.2017.07.014

Grassini, P., Eskridge, K. M., \& Cassman, K. G. (2013). Distinguishing between yield advances and yield plateaus in historical crop production trends. Nat. Commun., 4, 2918-2929. https://doi.org/10.1038/ ncomms 3918

Hao, W., Zhu, M. Z., Gao, J. P., Sun, S. Y., \& Lin, H. X. (2009). Identification of quantitative trait loci for rice quality in a population of chromosome segment substitution lines. J. Integr. Plant Biol., 51(5), 500-512. https://doi.org/10.1111/j.1744-7909.2009.00822.x

He, S. Q., Zhou, M. H., \& Dang, H. Y. (2014). Studies on optimal stubble height for Zhunliangyou 608 in planting pattern of main crop plus ratoon rice. Hybrid Rice, 29, 47-48.

Ichii, M., \& Kuwada, H. (1981). Application of a ratoon to a test of agronomic characters in rice breeding. I. Variation in ratoon ability and its relation to agronomic characters of mother plant. Japanese J. Breeding, 31(3), 273-278.

Ishimaru, T., Horigane, A. K., Ida, M., Iwasawa, N., San-oh, Y. A., Nakazono, M., ... Yoshida, M. (2009). Formation of grain chalkiness and changes in water distribution in developing rice caryopses grown under high-temperature stress. J. Cereal Sci., 50(2), 166-174. https://doi.org/10.1016/j.jcs.2009.04.011

Jin, J., Huang, W., Gao, J. P., Yang, J., Shi, M., Zhu, M. Z., ... Lin, H. X. (2008). Genetic control of rice plant architecture under domestication. Nat. Genet., 40, 1365-1369. https://doi.org/10.1038/ng.247

Krishnamurthy, K. (1988). Rice ratooning as an alternative to double cropping in Tropical Asia (pp. 3-15). Rice Ratooning IRRI, Manila, Philippines.

Laenoi, S., Rerkasem, B., Lordkaew, S., \& Prom-U-Thai, C. (2017). Seasonal variation in grain yield and quality in different rice varieties. Field Crops Res., 221, 350-357. https://doi.org/10.1016/j.fcr.2017.06.006

Li, N., Xu, R., Duan, P. G., \& Li, Y. H. (2018). Control of grain size in rice. Plant Reprod., 31(3), $237-251$. https://doi.org/10.1007/s00497-018-0333-6

Li, X. Y., Qian, Q., Fu, Z. M., Wang, Y. H., Xiong, G. S., Zeng, D. L., .. Li, J. Y. (2003a). Control of tillering in rice. Nature, 422(6932), 618-621. https://doi.org/10.1038/nature01518

Li, Y. B., Fan, C. C., Xing, Y. Z., Yun, P., Luo, L. J., Yan, B., ... He, Y. Q. (2014). Chalk5 encodes a vacuolar $\mathrm{H}(+)$-translocating pyrophosphatase influencing grain chalkiness in rice. Nat. Genet., 46(4), $398-404$. https://doi.org/10.1038/ng.2923

Li, Z. F., Wan, J. M., Xia, J. F., \& Zhai, H. Q. (2003b). Mapping quantitative trait loci underlying appearance quality of rice grains (Oryza sativa L.). Acta Genetica Sinica, 30(3), 251-259. https://doi.org/10.1016/ S0891-0618(02)00103-5

Liu, J. F., Chen, J., Zheng, X. M., Wu, F. Q., Lin, Q. B., Heng, Y. Q., .. Wan, J. M. (2017). GW5 acts in the brassinosteroid signalling pathway to regulate grain width and weight in rice. Nat. Plants, 3, 1199-1209. https://doi.org/10.1038/nplants.2017.43 
Lu, Y., Zhang, X. M., Qi, Y., Zhang, C. Q., Ling, Y. P., \& Liu, Q. Q. (2018). Scanning electron microscopic analysis of grain cross-section from rice with different transparency. Chin. J. Rice Sci., 32, 189-199.

Mao, H. L., Sun, S. Y., Yao, J. L., Wang, C. R., Yu, S. B., Xu, C. G., ... Zhang, Q. F. (2010). Linking differential domain functions of the GS3 protein to natural variation of grain size in rice. Proc. Natl. Acad. Sci., 107(45), 19579-19584. https://doi.org/10.1073/pnas.1014419107

Mei, H. W., Luo, L. J., Guo, L. B., Wang, Y. P., Yu, X. Q., Ying, C. S., \& Li, Z. K. (2002). Molecular mapping of QTLs for rice milling yield traits. Acta Genet Sin., 29(9), 791-797.

Moldenhauer, K. A. K., Gibbons, J. H., \& McKenzie, K. S. (2004). Rice varieties. In E. T. Champagne (Ed.), Rice: Chemistry and technology (3rd ed., pp. 49-75). The American Association of Cereal Chemists, St. Paul.

Nakano, H., \& Morita, S. (2007). Effects of twice harvesting on total dry matter yield of rice. Field Crops Res., 101, 269-275. https://doi.org/10.1016/j.fcr.2006.12.001

Nathaniel, D. M., James, S. G., Matt, J., Deepak, K. R., Navin, R., \& Jonathan A. F. (2012). Closing yield gaps through nutrient and water management. Nat., 490(7419), 254-257. https://doi.org/10.1038/nature11420

Nelson, J. C., Mcclung, A. M., Fjellstrom, R. G., Moldenhauer, K. A. K., Boza, E., Jodari, F., ... Yeater, K. M. (2011). Mapping QTL main and interaction influences on milling quality in elite us rice germplasm. Theor. Appl. Genet., 122(2), 291-309. https://doi.org/10.1007/s00122-010-1445-z

Oad, F. C., Cruz, P. S., Memon, N., Oad, N. L., \& Hassan, Z. U. (2002). Rice ratooning management. J. Appl. Sci., 2(1), 29-35. https://doi.org/10.3923/jas.2002.29.35

Peng, S. B., Huang, J. L., Sheehy, J. E., Laza, R. C., Visperas, R. M., Zhong, X. H., ... Cassman, K. G. (2004). Rice yields decline with higher night temperature from global warming. Proc. Natl. Acad. Sci., 101(27), 9971-9975. https://doi.org/10.1073/pnas.0403720101

Ray, D. K., \& Foley, J. A. (2013). Increasing global crop harvest frequency: Recent trends and future directions. Environ. Res. Lett., 8(4), 575-591. https://doi.org/10.1088/1748-9326/8/4/044041

Septiningsih, E. M., Trijatmiko, K. R., Moeljopawiro, S., \& McCouch, S. R. (2003). Identification of quantitative trait loci for grain quality in an advanced backcross population derived from the Oryza sativa variety IR64 and the wild relative O. rufipogon. Theor. Appl. Genet., 107(8), 1433-1441. https://doi.org/ $10.1007 / \mathrm{s} 00122-003-1373-2$

Song, X. J., Huang, W., Shi, M., Zhu, M. Z., \& Lin, H. X. (2007). A QTL for rice grain width and weight encodes a previously unknown RING-type E3 ubiquitin ligase. Nat. Genet., 39(5), 623-630. https://doi.org/ $10.1038 / \mathrm{ng} 2014$

Sun, X. H., Zhang, J. G., \& Liang, Y. J. (1988). Ratooning with rice hybrids (pp. 155-161). Rice ratooning IRRI, Manila, Philippines.

Tan, Y. F., Sun, M., Xing, Y. Z., Hua, J. P., Sun, X. L., Zhang, Q. F., \& Corke, H. (2001). Mapping quantitative trait loci for milling quality, protein content and color characteristics of rice using a recombinant inbred line population derived from an elite rice hybrid. Theor. Appl. Genet., 103(6-7), 1037-1045. https://doi.org/ $10.1007 / \mathrm{s} 001220100665$

Tashiro, T., \& Wardlaw, I. F. (1991). The effect of high temperature on kernel dimensions and the type and occurrence of kernel damage in rice. Aust. J. Agric. Res., 42, 485-496. https://doi.org/10.1071/ar9910485

Wan, Y. X., Deng, Q. M., Wang, S. Q., Liu, M. W., Zhou, H. Q., \& Li, P. (2007). Genetic polymorphism of $W x$ gene and its correlation with main grain quality characteristics in rice. Rice Sci., 14(2), 85-93. https://doi.org/10.1016/S1672-6308(07)60013-4

Wang, J., Lu, K., Nie, H. P., Zeng, Q. S., Wu, B. W., Qian, J. J., \& Fang, Z. M. (2018). Rice nitrate transporter OsNPF7.2 positively regulates tiller number and grain yield. Rice, 11(1), 12. https://doi.org/10.1186/ s12284-018-0205-6

Wang, S. K., Li, S., Liu, Q., Wu, K., Zhang, J. Q., Wang, S. S., ... Fu, X. D. (2015a). The OsSPL16-GW7 regulatory module determines grain shape and simultaneously improves rice yield and grain quality. Nat. Genet., 47(8), 949-954. https://doi.org/10.1038/ng.3352 
Wang, Y. X., Xiong, G. S., Hu, J., Jiang, L., Yu, H., Xu, J., ... Qian, Q. (2015b). Copy number variation at the GL17 locus contributes to grain size diversity in rice. Nat. Genet., 47(8), 944-948. https://doi.org/ 10.1038/ng.3346

Woo, M. O., Ham, T. H., Ji, H. S., Choi, M. S., Jiang, W., Chu, S. H., ... Koh, H. J. (2008). Inactivation of the UGPasel gene causes genic male sterility and endosperm chalkiness in rice (Oryza sativa L.). The Plant J., 54(2), 190-204. https://doi.org/10.1111/j.1365-313X.2008.03405.x

Xiong, H., Fang, W., \& Tan, Z. B. (1991). Effect of number of auxillary buds and main crop cutting time on ratoon crop yield. Int. Rice Res. News Lett., 16, 19.

Yi, Z. X., Zhou, W. X., \& Tu, N. M. (2009). Effects of stubble height of the main crop on source-sink characteristics and assimilates transportation in ratooning rice. Chin. J. Rice Sci., 23, 509-516.

Ying, J. F., Peng, S. B., He, Q. R., Yang, H., Yang, C. D., Visperas, R. M., \& Cassman, K. G. (1998). Comparison of high-yield rice in tropical and subtropical environments: I. Determinants of grain and dry matter yields. Field Crops Res., 57, 71-84. https://doi.org/10.1016/s0378-4290(98)00077-x

Zeng, D. L., Yan, M. X., Wang, Y. H., Liu, X. F., Qian, Q., \& Li, J. Y. (2007). Dul, encoding a novel Prp1 protein, regulates starch biosynthesis through affecting the splicing of $W x^{b}$ pre-mRNAs in rice (Oryza sativa L.). Plant Mol. Biol., 65(4), 501-509. https://doi.org/10.1007/s11103-007-9186-3

Zhang, C. Q., Zhu, L. J., Shao, K., Gu, M. H., \& Liu, Q. Q. (2013). Toward underlying reasons for rice starches having low viscosity and high amylose: Physiochemical and structural characteristics. J. Sci. Food Agric., 93(7), 1543-1551. https://doi.org/10.1002/jsfa.5987

Zhou, L. J., Chen, L. M., Jiang, L., Zhang, W. W., Liu, L. L., Liu, X., ... Wan, J. M. (2009). Fine mapping of the grain chalkiness QTL $q P G W C-7$ in rice (Oryza sativa L.). Theor. Appl. Genet., 118(3), 581-590. https://doi.org/10.1007/s00122-008-0922-0

\section{Copyrights}

Copyright for this article is retained by the author(s), with first publication rights granted to the journal.

This is an open-access article distributed under the terms and conditions of the Creative Commons Attribution license (http://creativecommons.org/licenses/by/4.0/). 